\title{
Desfecho da gravidez em portadoras de esclerodermia difusa e limitada
}

\author{
Pregnancy outcome in patients with diffuse and limited scleroderma
}

\author{
Marina Celli Francisco ${ }^{1}$, Felipe Trentin Neves ${ }^{2}$, Thelma Larocca Skare ${ }^{3}$, Ricardo Teodoro Beck ${ }^{4}$
}

\section{RESUM0}

Objetivo: verificar se existe diferença no número de abortamentos e recém-nascidos (RN) de baixo peso entre as pacientes com esclerodermia ( $\mathrm{SSc}$ ) se comparadas com as mulheres sem a doença entre as formas da doença e quando a manifestação da SSc ocorre antes ou após a gestação. Métodos: foram incluídas 26 pacientes com SSc. Os casos foram analisados quanto ao número de gestações, abortamentos, peso ao nascer e sexo dos filhos, correlacionando-se com a forma da doença e a relação temporal do diagnóstico com a gestação. As pacientes não haviam feito uso de medicações que interferissem na gestação, nem apresentavam outra doença. Para controle foram incluídas 26 mulheres saudáveis, sem doenças que alterassem o curso da gestação, pareadas para nível socioeconômico e idade. Para estudo estatístico foram feitas tabelas de freqüência, de contingência e empregados os testes de Fisher, $\chi^{2}$ e Mann-Whitney. Resultados: entre as pacientes com SSc, houve 96 gestações com 13,5\% $(\mathrm{n}=13)$ de abortamentos. No grupo controle, 94 gestações com 9,6\% $(\mathrm{n}=9)$ de abortamentos. Não houve diferença quanto ao número de $\mathrm{RN}$ de baixo peso nestes dois grupos $(\mathrm{n}=8$ e controles, $\mathrm{n}=6$, com $\mathrm{p}=0,54)$ e tampouco entre o número de abortos $(\mathrm{p}=0,46)$. Entretanto, o número de $\mathrm{RN}$ de baixo peso foi significativamente maior entre os casos com a forma difusa da doença (forma difusa, $n=4$ e limitada, $n=4$, com $p=0,04$ ) e em pacientes que engravidaram após o diagnóstico estabelecido de SSc (37,5\% em mulheres sabidamente doentes e 6,7\% em mulheres que engravidaram antes de adoecer, $\operatorname{com} \mathrm{p}=0,03)$. O número de $\mathrm{RN}$ masculinos foi maior no grupo das $\mathrm{SSc}(\mathrm{p}=0,002)$. Conclusões: as mulheres portadoras de SSc apresentam um número maior de RN de baixo peso na forma difusa da doença e quando a gravidez se estabelece após o diagnóstico clínico da doença.

PALAVRAS-CHAVE: Esclerodermia difusa; Aborto; Recém-nascido de baixo peso

\section{ABSTRACT}

Purpose: to verify if there is any difference in the number of miscarriages and newborns with low weight in patients with scleroderma (SSc) when compared with women without the disease, between the two clinical variants of the disease and when the expression of SSc occurred before or after the gestation. Methods: twenty-six patients were analyzed regarding the number of pregnancies, miscarriages, sex, and birth weight of the children, studying the clinical variant of the disease and the temporal relationship between diagnosis and gestation. The patients had not used either medications that could interfere in gestation or had any other disease that could do it. For control, twenty-six healthy women, without diseases that might alter the gestation, of the same ages and socioeconomic status were studied. For the statistical analysis study, tables of frequency, tables of contingency, and Fisher, $\chi^{2}$ and Mann-Whitney tests were used. Statistical significance was considered when $p<0.05$. Results: among the patients with SSc, there were 96 gestations with $13.5 \%(n=13)$ of miscarriages. In the control group, there were 94 gestations with $9.6 \%(n=9)$ of miscarriages. There were no differences in the number of newborns with low weight between the two groups (cases, $n=8$ and controls, $n=6$, with $p=0.54$ ), nor in the number of miscarriages $(\mathrm{p}=0.46)$. However, the number of newborn babies with low weight was significantly higher among the cases with the diffuse disease (diffuse form, $\mathrm{n}=4$ and limited, $\mathrm{n}=4$, with $\mathrm{p}=0.04$ ) and among patients that became pregnant after the diagnosis of SSc (37.5\% in women known to be sick and $6.7 \%$ in women who became pregnant before getting ill, with $\mathrm{p}=0.03)$. The number of male newborns was higher in the women with SSc $(\mathrm{p}=0.002)$. Conclusions: the women with SSc showed a higher number of low-weight newborns in the group with diffuse disease and when pregnancy occurred after the clinical diagnosis of the disease.

KEYWORDS: Scleroderma, diffuse; Abortion; Infant, low birth weight

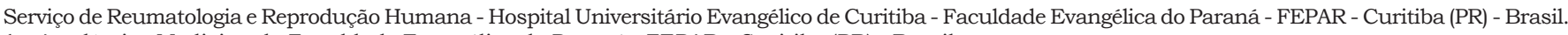
1 Acadêmico Medicina da Faculdade Evangélica do Paraná - FEPAR - Curitiba (PR) - Brasil.

2 Acadêmico Medicina da Faculdade Evangélica do Paraná - FEPAR - Curitiba (PR) - Brasil.

3 Professora da Disciplina de Reumatologia do Departamento de Clínica Médica da Faculdade Evangélica do Paraná - FEPAR - Curitiba (PR) - Brasil.

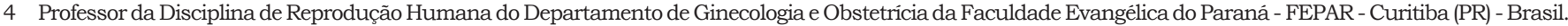
Correspondência: Felipe Trentin Neves

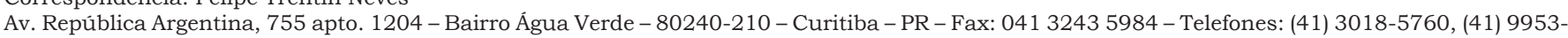

7793 - e-mail: felipetn $a$ terra.com.br 
Introdução

A esclerodermia sistêmica (SSc) é doença auto-imune grave, caracterizada por lesões fibrosas e vasculares afetando médios e pequenos vasos $^{1}$. Pele, pulmões, coração, rins e intestinos são os locais primários de dano e respondem pelas complicações da doença ${ }^{1}$. A fibrose pode ser causada por citocinas pró-fibróticas, como o fator de crescimento e transformação beta (TGF beta), interleucina 4, fator de crescimento derivado das plaquetas (PDGF) e o fator de crescimento de tecido conjuntivo. A vasculopatia pode ser causada pelo TGF beta e PDGF, ao passo que a escassez de vasos nas lesões da pele pode ser atribuída a anticorpos anticélulas endoteliais ${ }^{2}$.

Existem duas formas da doença: a forma difusa (dSSc) e a forma limitada (1SSc). Ambas acometem órgãos internos, todavia o que as diferencia é a localização das lesões cutâneas ${ }^{3}$.

A patogênese da SSc sistêmica persiste um enigma. Estudos genéticos, familiares e com gêmeos sugerem que a doença ocorre em individuos geneticamente suscetiveis ${ }^{4}$. A presença de determinados alelos de HLA exerce influência na predisposição a essa colagenose e na sua expressão ${ }^{5}$. Outro achado que pode estar implicado em sua gênese é a presença de microquimerismo, ou seja, o encontro de células fetais em tecidos maternos, estimulando o sistema auto-imune ${ }^{5,6}$.

A SSc afeta predominantemente o sexo feminino (3-8:1), sendo que esta relação alcança índices ainda maiores entre as idades de 15 e 50 anos (15:1). Tais achados sugerem que fatores hormonais possam contribuir com o aparecimento da doença ${ }^{7}$.

Relatos de gestação em pacientes com SSc são incomuns e isto pode ser explicado pela raridade da doença e pela sua gravidade, que faz com que as suas portadoras evitem a gravidez. O tema da associação entre fertilidade e SSc é dificil de ser discutido, visto que muitos fatores, tanto físicos quanto psicológicos, interferem na capacidade e no desejo de ficar grávida. Muitos médicos desaconselham mulheres com SSc a terem filhos, pois acreditam que exista aumento significante no número de abortos ${ }^{8}$. Com isto, dados existentes na literatura sobre fertilidade neste grupo de pacientes nem sempre são confiáveis.

Queixas como secura vaginal, dispareunia e irregularidades menstruais são bastante comuns em pacientes com esta colagenose. Todavia, poucas foram as anormalidades anatômicas encontradas. Anormalidades da cérvice uterina são raras e menopausa precoce foi relatada, no entanto sem comprovação estatística ${ }^{8}$.

$\mathrm{Na}$ gestante acometida por esclerodermia são observados aumento do risco de nascimento prematuro e retardo de crescimento intra-útero. Contudo, a ocorrência de índices elevados de abortos e óbitos perinatais apresenta dados conflitantes na literatu$\mathrm{ra}^{9,10}$. A etiopatogenia da morbidade fetal não é bem esclarecida. Uma meta-análise relatou que a presença de SSc confere maior risco de morte para as suas portadoras. O envolvimento de órgãos internos é importante fator que determina essa mortalidade ${ }^{11}$.

Os anticorpos anticardiolipina (aCL) fazem parte do grupo dos anticorpos antifosfolipídeos e estão presentes em muitas doenças auto-imunes, incluindo a SSc. Estes anticorpos apresentam correlação com trombose arterial e venosa, abortamentos de repetição, livedo reticular e trombocitopenia ${ }^{12-14}$. Tais manifestações dependem exclusivamente da presença desse tipo de anticorpo e não da doença subjacente, uma vez que os achados clínicos resultam das lesões isquêmicas em vários órgãos e não de vasculites. Deste modo, eventual taxa aumentada de abortos nessas pacientes poderia ter duas causas: a doença de base e/ou a presença desses anticorpos.

A mulher com SSc apresenta muitas dúvidas e angústias com relação a sua capacidade de engravidar e, se conseguir, qual a chance de esta gestação chegar a termo com um filho saudável. A maternidade é muito importante para a realização pessoal da maioria das mulheres, fato este que nos incentivou a executar este trabalho.

Este estudo foi feito com intuito de relatar a influência desta colagenose sobre a gravidez. Seu objetivo é verificar se as pacientes com esclerodermia apresentam maior número de abortos e recém-nascidos (RN) de baixo peso, se mulheres com a forma difusa da doença diferem nestes mesmos aspectos daquelas com a forma limitada e se a gestação em pacientes sabidamente com esclerodermia resulta em maior taxa de óbitos fetais e RN de baixo peso do que em pacientes sem a doença até o momento da gravidez.

\section{Pacientes e Métodos}

Foram incluídas 26 pacientes portadoras de esclerodermia, acompanhadas no ambulatório de Reumatologia do Hospital Universitário Evangélico de Curitiba durante o primeiro semestre de 2004 e residentes em Curitiba ou na região metropolitana. A idade minima era de 17 e a máxima de 62 anos (média de 40,2士12,9). O tempo de evolução da doença variava entre 1 e 34 anos (média de $6,8 \pm 7,8$ ).

Todas as pacientes preenchiam os critérios diagnósticos do American College of Rheumatology para $\mathrm{SSc}^{15}$. O critério maior para o diagnóstico é a presença de esclerodermia proximal no dorso das mãos. Por sua alta especificidade em separar esclerodermia de doenças similares, este foi selecionado como o único critério maior para a SSc, com $91 \%$ de sensibilidade e 99\% de especificidade. Os critérios menores são os seguintes: esclerodactilia, cicatrizes estelares nas 
polpas digitais e perda do coxim da porção distal do dedo e fibrose pulmonar bibasilar demonstrada na radiografia de tórax. Para o diagnóstico da doença é necessário um critério maior ou dois menores ${ }^{15}$.

As mulheres que possuíam SSc foram dividas em duas formas clínicas: dSSc e 1SSc, de acordo com os critérios de LeRoy et al. ${ }^{3}$, sendo 20 pacientes com $1 S S c$ e seis pacientes com dSSc. Os critérios de LeRoy et al. ${ }^{3}$ separam estas duas formas de acordo com o acometimento cutâneo, uma vez que ambas as formas podem afetar órgãos internos. A 1SSc é a forma da doença em que o envolvimento da pele é limitado às regiões distais aos cotovelos e joelhos, e superiores às clavículas. Já na dSSc qualquer região da pele pode ser acometida.

Nenhuma das pacientes era portadora de outra enfermidade que interferisse na concepção ou no curso da gravidez, nem havia feito uso de ciclofosfamida ou medicamentos que pudessem contra-indicar a gestação ou alterar a fertilidade.

As pacientes foram submetidas a um questionário-padrão, com perguntas quanto ao número de gestações, abortamentos, peso ao nascer e sexo dos filhos. A seguir, os prontuários foram revisados para estudo da presença dos anticorpos aCL.

Para controle, foram incluídas 26 mulheres saudáveis (funcionárias do hospital e acompanhantes de pacientes), pareadas para nível socioeconômico, idade e renda, as quais foram submetidas ao mesmo questionário. Para sua seleção, foram utilizados os seguintes critérios: ser usuária do SUS; ter idade e renda (em número de salários mínimos/mês) semelhantes aos do grupo com esclerodermia; não apresentar doença de base que pudesse causar dificuldades na concepção e na manutenção da gestação; sem doenças crônicas. Deveriam também ser moradoras de Curitiba ou região.

Verificou-se o número de gestações e de abortos nos dois grupos, bem como o número de RN vivos, o número de RN de baixo peso (peso $<2.490 \mathrm{~g}$ ) e o número de RN do sexo masculino. Esses mesmos dados foram também estudados nas duas formas de SSc (dSSc e 1SSc). Analisou-se, ainda, a diferença do número de abortamentos e RN de baixo peso entre as pacientes com gestação antes do diagnóstico de SSc e aquelas com gestação após o diagnóstico. Por último, foi verificado o número de abortos e de nascidos vivos nas pacientes com aCL positivo.

Para estudo estatístico foram feitas tabelas de freqüência e de contingência, além de testes de Fisher e $\chi^{2}$ para variáveis como positividade de anticorpos aCL e prevalência de sexo dos RN. O teste de Mann-Whitney foi usado para variáveis contínuas, como comparação entre número de gestações, número de abortamentos e peso do $\mathrm{RN}$ ao nascer entre os diferentes grupos. Foram considerados significantes valores de $\mathrm{p}$ menores que 0,05.

Este estudo foi aprovado pelo Comitê de Ética em Pesquisa da Faculdade Evangélica do Paraná.

\section{Resultados}

Entre as 26 portadoras de esclerodermia, houve 96 gestações com 83 nascidos vivos e 13 abortamentos $(13,5 \%)$. Cinco pacientes $(19,2 \%)$ eram nuliparas, porém nenhuma delas apresentava história confirmada de infertilidade. No grupo controle houve 94 gestações com 85 (94,4\%) nascidos vivos, nove abortamentos $(9,6 \%)$ e duas pacientes nuliparas.

$\mathrm{O}$ número de nascidos vivos variou de 0 a 13 nas esclerodérmicas e de 0 a 11 no grupo controle, com mediana três em ambos os grupos. Não houve diferença significativa no número de $\mathrm{RN}$ de baixo peso $(p=0,54)$, quando se comparou o grupo portador da colagenose apresentado ( $n=8$ ou $8,3 \%$ ) e o grupo controle ( $n=6$ ou $6,4 \%$ ). A média do peso ao nascer nos dois grupos também não diferiu significativamente $(p=0,426)$. O número de RN do sexo masculino foi significativamente maior no grupo das esclerodérmicas ( $n=46$ ou $47,9 \%$ ) com relação às pacientes controles ( $\mathrm{n}=32$ ou $34 \%$ ), com $\mathrm{p}=0,002$. Estes dados estão resumidos na Tabela 1.

Tabela 1 - Número de abortamentos, recém-nascidos (RN) de baixo peso e RN do sexo masculino entre as pacientes com esclerodermia comparadas com grupo controle.

\begin{tabular}{lccc}
\hline & $\begin{array}{c}\text { Pacientes com } \\
\text { esclerodermia } \\
n=26\end{array}$ & $\begin{array}{c}\text { Controles } \\
\mathbf{n}=\mathbf{2 6}\end{array}$ & $\mathbf{p}$ \\
\hline Número de gestações (total) & $\mathrm{n}=96$ & $\mathrm{n}=94$ & - \\
Número de gestações/paciente & $0-13$ (mediana $=3)$ & $0-11$ (mediana $=3)$ & 0,87 \\
Número de abortamentos & $\mathrm{n}=13(13,5 \%)$ & $\mathrm{n}=9(9,6 \%)$ & 0,46 \\
Número de RN de baixo peso & $\mathrm{n}=8(8,3 \%)$ & $\mathrm{n}=6(6,4 \%)$ & 0,54 \\
RN do sexo masculino & $\mathrm{n}=46(47,9 \%)$ & $\mathrm{n}=32(34 \%)$ & 0,002 \\
\hline
\end{tabular}

$\mathrm{n}=$ número da amostra.

Entre as pacientes com SSc, 20 apresentavam a forma $1 \mathrm{SSc}$ e seis, a forma dSSc. As pacientes com 1SSc tinham tido 77 gestações, com nove abortamentos $(11,7 \%)$ e 68 nascidos vivos $(88,3 \%)$. Já as pacientes com dSSc tinham 19 gestações, com quatro abortamentos (21\%) e 15 nascidos vivos. Não houve diferença quanto ao número de abortamentos entre as duas formas de SSc $(\mathrm{p}=0,28)$. O número de RN de baixo peso foi significativamente maior no grupo com a forma difusa ( $\mathrm{n}=4$ ou $26,7 \%$ ) do que naquele com a forma limitada ( $\mathrm{n}=4$ ou $5,9 \%$ ), com $\mathrm{p}=0,04$ (Tabela 2 ).

Tabela 2 - Comparação entre as formas de esclerodermia limitada e difusa, quanto ao número de abortamentos, recém-nascido (RN) de baixo peso e presença do anticorpo anticardiolipina.

\begin{tabular}{lccc}
\hline & $\begin{array}{c}\text { Esclerodermia } \\
\text { limitada } \\
n=20\end{array}$ & $\begin{array}{c}\text { Esclerodermia } \\
\text { difusa }\end{array}$ & $\mathbf{p}$ \\
\hline Número de gestações & $n=6$ & \\
Número de abortamentos & $n=9(11,7 \%)$ & $n=4(21 \%)$ & 0.28 \\
Número de RN de baixo peso & $n=4(5,9 \%)$ & $n=4(26,7 \%)$ & 0.04 \\
Ac anticardiolipina positivo & $n=05$ & $n=0$ & - \\
\hline $\mathrm{n}=$ número da amostra; Ac = anticorpo. & & &
\end{tabular}


Nas pacientes acometidas por SSc, 86 gestações aconteceram antes do aparecimento da doença e 10 ocorreram depois. Das gestações ocorridas antes da manifestação da doença, $11(12,8 \%)$ resultaram em abortamento, contra duas $(20 \%)$ depois da manifestação da mesma, dado esse sem significância estatística $(p=0,29)$. Em nenhuma paciente a SSc surgiu durante a gestação. Nesses dois grupos também ocorreu uma diferença significativa no número de $R N$ de baixo peso $(p=0,03)$, sendo esse número maior nas pacientes que engravidaram depois da manifestação da doença ( $\mathrm{n}=3$ ou $37,5 \%$ ), contra as pacientes com gestações antes da manifestação da doença ( $n=5$ ou $6,7 \%$ ). Os dados referentes a esta análise estão na Tabela 3.

Tabela 3 - Taxa de abortamentos e recém-nascido (RN) de baixo peso em pacientes com gestação anterior e posterior a esclerodermia.

\begin{tabular}{llll}
\hline & $\begin{array}{c}\text { Gestações antes } \\
\text { do diagnóstico } \\
n=86\end{array}$ & $\begin{array}{c}\text { Gestações depois } \\
\text { do diagnóstico }\end{array}$ & $p$ \\
& $n=10$ & \\
\hline Abortamento & $n=11(12,8 \%)$ & $n=2(20 \%)$ & 0.29 \\
RN de baixo peso & $n=5(6,7 \%)$ & $n=3(37,5 \%)$ & 0.03 \\
\hline$n=$ número da amostra & & &
\end{tabular}

Cinco pacientes com a esclerodermia apresentavam anticorpos aCL positivos, sendo todas elas da forma limitada da doença. Nessas pacientes não se encontrou número de abortos maior que na população esclerodérmica com aCL negativo $(\mathrm{p}=0,17)$.

\section{Discussão}

A SSc tem incidência de aproximadamente 1,10 casos para cada 100.000 habitantes, de acordo com um estudo populacional grego ${ }^{16}$. Porém, essa incidência pode estar subestimada, uma vez que o estudo considerou em sua casuística apenas pacientes que estavam em acompanhamento com reumatologistas.

A SSc é doença rara que afeta predominantemente o sexo feminino, entre a terceira e a quinta décadas ${ }^{7}$. Em nosso estudo confirmamos este dado, tendo encontrado uma média de idade próxima aos 34 anos. Sugere-se que a predominância da doença nesta idade está relacionada ao padrão hormonal ${ }^{17}$. Devido à maior prevalência da SSc nesta faixa etária, calcula-se que pelo menos metade das mulheres com esclerodermia ficarão grávidas após o aparecimento da sua doença ${ }^{8}$.

Apenas 2 a $5 \%$ das mulheres com SSc que tentaram engravidar não obtiveram sucesso e a porcentagem de mulheres que levaram pelo menos um ano para engravidar não foi diferente do restante da população ${ }^{8}$. Entretanto, neste estudo não se separa- ram os casos conforme a presença de aCL, nem pelo uso prévio de imunossupressores que pudessem interferir com a concepção. Em nossa casuística cinco pacientes com SSc não tiveram filhos, porém apenas uma havia tentado a concepção, sendo que não houve investigação de infertilidade no casal.

Alguns estudos caso-controle identificaram freqüência aumentada de abortos por gestações ${ }^{18}$, mesmo em mulheres que engravidaram antes da manifestação da doença ${ }^{19}$. Em outro, porém, no qual foram incluídas 48 pacientes com SSc, não se observou diferença no número de abortos de pacientes com SSc, artrite reumatóide e controles ${ }^{10}$. Um estudo grande (caso-controle) com 214 mulheres aponta para o mesmo sentido ${ }^{8}$.

No que se refere a uma maior ocorrência de abortamentos conforme a forma da doença, um estudo com 150 pacientes, este de origem nacional, mostrou que a forma da esclerodermia não interfere na prevalência de abortamentos ${ }^{7}$. Entretanto, neste último, comparou-se apenas a diferença em portadoras da doença nas suas duas formas e a relação temporal da gestação com a manifestação clínica da doença.

No presente estudo, encontramos taxa de aborto de $13,5 \%$ nas pacientes com SSc, a qual não foi significativamente maior que a do grupo controle. Também não encontramos diferenças no número de abortamentos por pacientes entre as mulheres com diagnóstico da doença antes e depois das gestações, nem entre as formas limitada e difusa.

Quanto à taxa de RN de baixo peso em mulheres com SSc, a literatura mostra que esta foi superior a da população em geral (10 versus $2 \%)^{10}$ e que a freqüência aumentada de RN a termo de baixo peso incide apenas nas pacientes com gestação após o aparecimento da doença ${ }^{20}$.

Não observamos diferença significativa entre o número de RN de baixo peso de pacientes com SSc e controles. Todavia, houve número significativamente maior de RN de baixo peso nas pacientes com SSc anterior à gestação se comparadas àquelas com diagnóstico posterior. O mesmo ocorreu entre as pacientes com forma difusa em relação às com a forma limitada.

Estudos com técnicas de biologia molecular mostram que ocorre troca celular entre a mãe e o feto durante uma gestação normal e que as células fetais podem sobreviver nos tecidos e sangue materno por muitos anos após a gestação ${ }^{21}$. A este fenômeno é dado o nome de microquimerismo e ele tem sido envolvido hipoteticamente na patogênese de algumas doenças auto-imunes, principalmente $\mathrm{SSc}^{6,17,22}$. Muitos criticam essa hipótese, alegando que o fenômeno também ocorre em pacientes saudáveis. No entanto, relatouse que pacientes com esta colagenose apresentam concentração de DNA masculino no sangue significativamente maior que na população normal ${ }^{17}$. Seqüências 
de DNA, específicas do cromossomo Y, têm sido achadas em altas concentrações nas células mononucleares sangüineas periféricas e lesões de pele de mulheres com $\mathrm{SSc}^{17}$. Seguindo estes dados, analisamos a freqüência de filhos homens e encontramos valores superiores nas pacientes com a doença se comparadas ao grupo controle.

$\mathrm{O}$ aCL é um anticorpo antifosfolipídeo que pode estar presente na SSc em taxas que variam entre 0 e $63 \%$ e sua presença parece não depender da forma de doença ${ }^{13}$. Tal anticorpo merece análise, neste contexto, por estar implicado em maiores taxas de abortamento. Em nosso estudo, 19,2\% das pacientes apresentavam aCL positivo, sendo todas elas com forma limitada da doença. Não encontramos diferença ao correlacionarmos esses anticorpos com história prévia de aborto ou trombose. Entretanto, entre as pacientes positivas para este anticorpo encontravam-se duas das que nunca tiveram filhos, o que prejudica esta análise.

Concluindo, pode-se dizer que a taxa de abortos e de RN de baixo peso nas pacientes com SSc não foi significativamente diferente da população em geral.

Ao serem comparadas as diferentes formas de apresentação da SSc, encontrou-se que o número de RN de baixo peso foi significativamente maior entre os casos com a forma difusa, embora, em relação a abortos, as duas formas tiveram o mesmo comportamento.

\section{Referências}

1. Lambe M, Bjornadal L, Neregard P, Nyren O, Cooper GS. Childbearing and the risk of scleroderma: a population-based study in Sweden. Am J Epidemiol. 2004;159(2):162-6.

2. Sakkas LI. New developments in the pathogenesis of systemic sclerosis. Autoimmunity. 2005;38(2):113-6.

3. LeRoy EC, Black C, Fleischmajer R, Jablonska S, Krieg T, Medsger TA Jr, et al. Scleroderma (systemic sclerosis): classification, subsets and pathogenesis. J Rheumatol. 1988;15(2):202-5.

4. Feghali-Bostwick CA. Genetics and proteomics in scleroderma. Curr Rheumatol Rep. 2005;7(2):129-34.

5. Gladman DD, Kung TN, Siannis F, Pellett F, Farewell VT, Lee P. HLA markers for susceptibility and expression in scleroderma. J Rheumatol. 2005;32(8):1481-7.

6. Lambert NC, Erickson TD, Yan Z, Pang JM, Guthrie KA, Furst DE, et al. Quantification of maternal microchimerism by HLA-specific real-time polymerase chain reaction: studies of healthy women and women with scleroderma. Arthritis Rheum. 2004;50(3):906-14.

7. Sampaio-Barros PD, Samara AM, Marques Neto JF. Gynaecologic history in systemic sclerosis. Clin Rheumatol. 2000;19(3):184-7.
8. Steen VD. Scleroderma and pregnancy. Rheum Dis Clin North Am. 1997;23(1):133-47.

9. Valentini G, Black C. Systemic sclerosis. Best Pract Res Clin Rheumatol. 2002;16(5):807-16.

10. Steen VD, Conte C, Day N, Ramsey-Goldman R, Medsger TA Jr. Pregnancy in women with systemic sclerosis. Arthritis Rheum. 1989;32(2):151-7.

11. Ioannidis JP, Vlachoyiannopoulos PG, Haidich AB, Medsger TA Jr, Lucas M, Michet CJ, et al. Mortality in systemic sclerosis: an international metaanalysis of individual patient data. Am J Med. 2005;118(1):2-10.

12. Picarelli MM, Staub HL, Bueno D, Pinto ME, Petracco A, Keiserman MW. Anticardiolipin antibodies in patients with recurrent abortions. Rev Bras Reumatol. 1995;35(4):189-92.

13.Pope JE, Thompson A. The frequency and significance of anticardiolipin antibodies in scleroderma. J Rheumatol. 2000;27(6):1450-2.

14. Mulinari-Brenner FA, Dal Pizzol AS, Thomaz PCP, Santamaría JR, Gemelli JJ. Antiphospholipid antibody syndrome. An Bras Dermatol. 2001;76(5):605-10.

15. Preliminary criteria for the classification of systemic sclerosis (scleroderma). Subcommittee for scleroderma criteria of the American Rheumatism Association Diagnostic and Therapeutic Criteria Committee. Arthritis Rheum. 1980;23(5):581-90.

16. Alamanos Y, Tsifetaki N, Voulgari PV, Siozos C, Tsamandouraki K, Alexiou GA, et al. Epidemiology of systemic sclerosis in northwest Greece 1981 to 2002. Semin Arthritis Rheum. 2005;34(5):714-20.

17. Pisa FE, Bovenzi M, Romeo L, Tonello A, Biasi D, Bambara LM, et al. Reproductive factors and the risk of scleroderma: an Italian case-control study. Arthritis Rheum. 2002;46(2):451-6.

18. Englert H, Brennan P, McNeil D, Black C, Silman AJ. Reproductive function prior to disease onset in women with scleroderma. J Rheumatol. 1992;19(10):1575-9.

19. Siamopoulou-Mavridou A, Manoussakis MN, Mavridis AK, Moutsopoulos HM. Outcome of pregnancy in patients with autoimmune rheumatic disease before the disease. Ann Rheum Dis. 1988;47(12):982-7.

20. Steen VD, Medsger TA Jr. Fertility and pregnancy outcome in women with systemic sclerosis. Arthritis Rheum. 1999;42(4):763-8.

21. Khosrotehrani K, Johnson KL, Lau J, Dupuy A, Cha DH, Bianchi DW. The influence of fetal loss on the presence of fetal cell microchimerism: a systematic review. Arthritis Rheum. 2003;48(11):3237-41.

22. Burastero SE, Galbiati S, Vassallo A, Sabbadini MG, Bellone M, Marchionni L, et al. Cellular microchimerism as a lifelong physiologic status in parous women: an immunologic basis for its amplification in patients with systemic sclerosis. Arthritis Rheum. 2003;48(4):1109-16. 